\title{
Awareness of HPV Infection and Vaccination among Teens in Urban High School
}

\author{
Faith C. Diorgu ${ }^{\text {1* }}$ and Kelechim N Diorgu ${ }^{2}$ \\ ${ }^{1}$ Nurse/Midwife lecturer, PhD, RM, Department of Nursing Science, University of Port Harcourt, Nigeria \\ ${ }^{2}$ Oncology clinical research associate, Sarah Cannon research institute, Nashville, TN 37203, USA \\ ${ }^{*}$ Corresponding Author: Nurse/Midwife lecturer, PhD, RM, Department of Nursing Science, University of Port Harcourt, Nigeria; Tel: +2348033401555; \\ E-mail: faith.diorgu@uniport.edu.ng
}

Received: March 06, 2019; Accepted: March 20, 2019; Published: April 20, 2019;

\begin{abstract}
To assess the level of awareness and knowledge of HPV infection and vaccination among 648 Teens from secondary schools and colleges in four metro cities of Port Harcourt Nigeria.

Materials and methods: This cross-sectional study was conducted by from 1 ${ }^{\text {st }}$ March to 31st August, 2018. Girls of 13-19 years, with an average of 16 years are targeted. A written questionnaire with two parts has been applied. A preliminary written questionnaire included questions of HPV infection and vaccination awareness.
\end{abstract}

Results: The study participants are poorly aware about HPV infection and vaccination but are intensely willing to know about it. 98\% (n-632) are not aware of HPV infection, while, 98\% (n-636) is not aware of the vaccination.

Conclusion: This study brings out the unawareness about HPV infection and vaccination in urban adolescent in four metro cities in Port Harcourt Nigeria. Adolescent understanding and being aware of the HPV virus is needed to have successful vaccination programs in Nigeria.

Keywords: Adolescents and HPV, Cancer cervix, HPV Awareness, HPV Vaccination

\section{Background}

Human Papillomavirus (HPV) is a common virus reported to be responsible to some type of cancers. HPV infection is the major risk factor for cervical cancer [1]. The prevalence rate of more than $85 \%$ global cervical cancer occurs in developing countries. This health burden is a public health issue that could be prevented by early vaccination of adolescents against HPV. A prophylactic vaccination against the HPV has the ability to reduce HPV infection and cervical cancer occurrence, thereby saving HPV many lives [2]. HPV vaccine approved by FDA has been in circulation since the year 2006 [3]. Notwithstanding, HPV vaccine implementation is still facing challenges in acceptance and lack of awareness despite its futuristic benefits. Awareness has been found to be low in some population and high in another $[4,5]$, but studies are yet to investigate the awareness of teens in Nigeria, If one exist, it does not measure the awareness of both the HPV infection and HPV vaccination of teenagers in high school. To enable practical programmed of HPV vaccination in the future, it will be required to assess the awareness and knowledge regarding HPV and vaccination among the teens population. If awareness could influence the acceptability of the uptake of the vaccination, it may then be wise to target public awareness as commonly done [6]. More research focus should be targeted on adolescent population as they constitute the interest group. This study therefor, explores the teens urban dwellers' awareness and knowledge about HPV infection and it vaccination.
Methods

\section{Design}

A longitudinal cohort study design with a cross sectional quantitative analysis. The study assessed baseline awareness and knowledge of HPV infection and HPV vaccination among teens in a four selected High School in Port Harcourt, Nigeria. The study was conducted from $1^{\text {st }}$ March to $31^{\text {st }}$ August, 2018.

\section{Study population and Recruitment}

For the teens studied, the eligibility criteria included ages $13-19$ years in High School. The method of selection and sampling units (high school) was through random. Four urban High Schools were purposively selected. Six hundred and forty eight (648) Teens were then randomly selected from the four high schools.

\section{Data Collection}

Each respondent completed a consent form and a questionnaire. Participation was voluntary and anonymous. Selected Teens were assembled in a room on the day of the survey. Each respondent filled the questionnaire under close supervision and the purpose and procedure of the survey explained. Data was collected, starting from the first school to the 4 th school. The research questionnaire consisted of two parts; social demographic profile, and awareness questions 
form the second part. The demographic data included information regarding sexual activity. This was done in other to predict the sexual behavior risk factor to HPV infection.

\section{Result and Statistical Analysis}

In this study, simple descriptive statistics were used. The study question items were organized into categories: demographic 5 items, awareness based 8 items which include awareness of HPV infection and awareness of HPV vaccination. Questions were answered in "Yes" or 'NO' items. Those with answers "Yes" = 1 point and "No" $=0$ point. All scores were summed up to calculate the overall awareness scores for HPV infection and HPV vaccination.

The study considered 648 Teens between the ages of 13 to 19 years, with 16 years as mean age. The rationale for the sample size and sampling is to see if there are any significant gaps between the level of awareness on HPV infection and HPV vaccination and between the girls and boys participants (Table 1). Of the study population (n$648)$ Teens, $41 \%(n-264)$ were boys and $59 \%$ (n- 384) were girls. The preliminary questionnaire assessed the awareness. Awareness was low. Only $2 \%(n-16)$ were aware of HPV infection, and 98\% ( $n=632)$ were not. Also 2\% (n - 12) knew about HPV vaccination and 98\% (n636) did not know. Out of 12 and 16 participants that had heard about HPV infection and HPV vaccination respectively, $1 \%(\mathrm{n}-3)$ were boys and $3 \%(n-13)$ were girls. Majority of both boys and girls were not aware. Few of the participants that were aware heard about HPV from their parents and friends (Table 2).

Table 1. The demographic data of the study population.

\begin{tabular}{|l|l|l|l|}
\hline \multicolumn{1}{|c|}{ Variable } & \multicolumn{1}{|c|}{ Classification } & \multicolumn{1}{c|}{ Frequency } & \multicolumn{1}{c|}{ Percentage } \\
\hline Age & $9-14$ & 432 & $67 \%$ \\
& $15-19$ & 216 & $33 \%$ \\
\hline Sex & Boys & 264 & $41 \%$ \\
& Girls & 384 & $59 \%$ \\
\hline Have had sex & Yes & 182 & $28 \%$ \\
& No & 466 & $72 \%$ \\
\hline Age at first sex & 13 years & 7 & $4 \%$ \\
& 14 years & 22 & $12 \%$ \\
& 15 years & 48 & $26 \%$ \\
& 16 years and above & 105 & $58 \%$ \\
\hline
\end{tabular}

\section{Discussion}

The study found result not consistent with some previous studies on high school students' knowledge and awareness of HPV infection and vaccination $[7,8]$. It was anticipated that there would be unawareness across participants as there is no knowledge programmes in schools and colleges regarding HPV and its vaccination. Nigeria adolescences seem to be disadvantaged group both economically and by their lack of knowledge and health awareness like many other
African countries. However the findings of this study may have limited generalizability to Nigerians with cultural diversity, different religions, and socioeconomic status that are distinctively different from western societies. Adequate knowledge and awareness of the clinical health importance of HPV infection and vaccination usually provide positive influence.

Table 2. Awareness on HPV Infection and HPV Vaccination and between boys and girl.

\begin{tabular}{|l|l|c|c|}
\hline \multicolumn{1}{|c|}{ Awareness } & Classification & Frequency & Percentage \\
\hline HPV Infection & No Awareness & 632 & $98 \%$ \\
& Yes Awareness & 16 & $2 \%$ \\
\hline HPV Vaccination & No Awareness & 636 & $98 \%$ \\
& Yes Awareness & 12 & $2 \%$ \\
\hline Boys & Yes Awareness & 3 & $1 \%$ \\
& No Awareness & 261 & $99 \%$ \\
\hline Girls & Yes Awareness & 13 & $3 \%$ \\
& No Awareness & 381 & $97 \%$ \\
\hline
\end{tabular}

In addition, when comparing the social and demographic characteristics among the respondents from four schools based on their gender, the differences were rather small, majority of participants had little knowledge and awareness on HPV infection or HPV vaccination across the four schools in the study. Researchers from west found out that adolescents who have high knowledge levels about HPV and cervical cancer, their acceptance of vaccine is also high [9]. Ninety nine percent girls and $97 \%$ boys have not heard of HPV virus and but were willing to know more about the infection. The new option of HPV vaccine as a primary prevention in adolescents is a unique opportunity of this era which promises a significant reduction of cervical cancer in the coming decades [10]. More efforts are needed to provide adolescence with information which will help them to be excluded from among those thousands of women who die from this preventable condition.

\section{Conclusion}

This study brings out the unawareness about HPV infection and vaccination in urban Teens in 4 High schools in Port Harcourt city, Nigeria. The study participants are poorly aware about HPV infection and vaccination but are intensely willing to know about them. In conclusion, findings of this study suggest that HPV infection and vaccination are not likely to encourage adolescent sexual activity. In addition, efforts should be made to increase knowledge-based programs in schools and colleges.

\section{Acknowledgement}

The authors gratefully acknowledged Dr. C. TobI-West, and Dr. EO. Oranu for their contributions. I also acknowledge all the high school students who participated. 


\section{Authors Contributions}

FCD had primary responsibility for protocol development, data collection, analysis and writing of the paper. KND performed final data analysis, participated in the development of the protocol and analytical framework for the study and contributed to the writing of the study.

\section{What this study Add}

It raised alarm on the poor awareness on HPV infection and Vaccination. It also highlight that the relevant group for HPV vaccination are willing to receive the vaccine.

\section{References}

1. Cohen J (2005) Public health. High hopes and dilemmas for a cervical cancer vaccine. Science 308: 618-621. [crossref]

2. Smith JS, Lindsay L, Hoots B (2007) Human papillomavirus type distribution in invasive cervical cancer and high-grade cervical lesions: a meta-analysis update. Int J Cancer 121: 621-632

3. Walsh CD, Gera A, Shah M (2008) Public knowledge and attitudes towards human papilloma virus (HPV) vaccination. BMC Public Health 8: 368.

4. Fu LY, Bonhomme LA, Cooper SC, Joseph JG, Zimet GD (2014) Educational interventions to increase HPV vaccination acceptance: a systematic review. Vaccine 32: $1901-1920$

5. Small SL, Sampselle CM, Martyn KK (2013) Dempsey AF. Modifiable influences on female HPV vaccine uptake at the clinic encounter level: a literature review. $J$ Am Assoc Nurs Pract [published online ahead of print August 22, 2013].

6. Blasi PR, King D, Henrikson NB (2015) HPV Vaccine Public Awareness Campaigns: An Environmental Scan. Health Promot Pract 16: 897-905. [crossref]

7. Jill B, Melissa KF, Michael JW Jr (2012) Adolescent understanding and acceptance of the HPV vaccination in an underserved population in New York City. J Oncol 2012, Article ID 904034.

8. Ericka C (2001) Lambert-college students' knowledge of human papillomavirus and effectiveness of a brief educational intervention. J Am Board Fam Pract 14: $178-183$.

9. Marlow LA, Waller J, Wardle J (2007) Public awareness that HPV is a risk factor for cervical cancer. Br J Cancer 97: 691-694. [crossref]

10. Brabin L, Roberts SA, Henry CK (2007) A semi-qualitative study of attitudes to vaccinating adolescents against HPV without parental consent-BMC Public health 2007. http://www.biomedcentral.com/1471-2458/7/20.

\section{Citation:}

Faith C. Diorgu and Kelechim N Diorgu (2019) Awareness of HPV Infection and Vaccination among Teens in Urban High School. ARCH Women Health Care Volume 2(2): 1-3. 